\title{
Isolated Dorsal Dislocation of the Lunate
}

\author{
N.A. Siddiqui ${ }^{*}, 1$ and S.P. Sarkar ${ }^{2}$ \\ ${ }^{I}$ Upper Limb Unit, Department of Orthopaedics, Princess Alexandra Hospital, Woolloongabba, Brisbane, Queensland \\ 4102, Australia \\ ${ }^{2}$ Department of Trauma and Orthopaedics, Southend University Hospital NHS Foundation Trust, Westcliff-on-Sea, \\ Essex SSO ORY, UK
}

\begin{abstract}
Lunate dislocations are well described in the volar direction as part of the perilunate dislocation, sometimes together with fractures of the other carpal bones or distal radius, as described by the anatomical studies of Mayfield [1]. It is a result of disruption of the complex inter-carpal and radio-carpal ligaments that hold the well conforming carpus in their normal position. Given the strength of these structures a significant trauma is required to cause them to fail.

However, we present a case of a patient who not only presented with relatively trivial trauma that resulted in a lunate dislocation, but it was also in the dorsal direction and not associated with any fracture or neurological compromise. In addition, she presented several days after her injury.

We treated her with closed manipulation and percutaneous K-wire fixation followed by a short period of immobilisation in a Plaster-of-Paris cast, with rapid return to full duties at work.

As many volar lunate dislocations may be missed at presentation, we suggest that in patients with relatively trivial trauma there should also be a suspicion of the lunate dislocating dorsally, which may be treated successfully without the aggressive open surgery usually required in volar perilunate dislocations.
\end{abstract}

Keywords: Carpus, dislocation, dorsal, ligament rupture, lunate, perilunate.

\section{INTRODUCTION}

Carpal dislocations are rare, and usually a result of significant energy such as a fall on the outstretched hand or road traffic accidents. There is disruption of the strong radiocarpal and inter-carpal ligaments. The commonest pattern of carpal dislocation is the perilunate dislocation, where failure of the restraining structures results in first a dorsal dislocation of the carpus and then reduction of the carpus back onto the radius propelling the lunate volarly. Dislocation of the lunate has also been associated with a fracture of one or more of the carpal bones, particularly the scaphoid. Dislocation of the lunate is usually in the volar direction [1]. However, dorsal dislocation of just the lunate is extremely rare, with reports in the literature usually associated with concomitant fractures of other carpal bones or even the distal radius.

We present a case report of isolated dorsal dislocation of the lunate with no concomitant fractures, as a result of a relatively low energy injury. This was treated with closed reduction and K-wire fixation with a short period in plaster cast immobilisation. With early K-wire removal and only a short time in plaster cast immobilisation the patient was able to return to full duties in their normal occupation within three months of surgery.

*Address correspondence to this author at the Upper Limb Unit, Department of Orthopaedics, Princess Alexandra Hospital, Woolloongabba, Brisbane, Queensland 4102, Australia; Tel: +61 73176 6106; Fax: +61 73176 5156; E-mail: nashat75@gmail.com

\section{PATIENT HISTORY}

The patient is a 56 year old female high school teacher who is right hand dominant, presenting with pain in her left wrist. Her injury was the result of a sudden pull by her dog whilst she was holding the lead with her left hand. She managed to maintain her balance and did not fall. Pain was immediate, followed by swelling within a few hours. She was able to continue teaching for two weeks with moderate pain around her left wrist, and had to limit activities that required both hands. She was taking infrequent simple analgesia. Eventually she had to attend her local Emergency Department due to persistent pain. She was initially thought to have a perilunate dislocation by staff in the Emergency Department and was referred to the Orthopaedic on-call team for further management.

On examination there was diffuse swelling around the dorsum of her wrist with mild erythema. A firm mass approximately $1 \mathrm{~cm}$ in diameter was easily palpable on the dorsum of the left wrist in the region of the lunate, which was mildly tender. The mass did not move with wrist movements. With firm pressure and longitudinal traction on the fingers the mass reduced but then instantly reappeared with cessation of traction and any movement of the wrist. The reduction manoeuvre was painful for the patient, but painless when the dorsal mass reappeared.

She had a global restriction of wrist movements compared to her unaffected side. Wrist flexion/extension was 20 degrees in either direction. She had full pronation but 
supination was reduced by 20 degrees compared to her normal side. Radial and ulnar deviation were both painful, being only 15 degrees each. She had moderately reduced grip strength and no restriction in movement of her digits. Pincer grip produced slight pain but she had normal power. Kirk-Watson test was too painful to perform.

She had normal sensation to the hand with no sign of intrinsic muscle wasting or weakness. She had good radial and ulnar pulses with capillary refill under 2 seconds. She had not suffered any previous injuries to this wrist, and other than some occasional pain around the thumb carpometacarpal joint had never experienced any problems with pain, function, instability, locking, or clicking. Her overall health was good, with only well controlled hypertension as her co-morbidity.

Plain radiographs (Figs. 1, 2) show the lunate dislocated dorsally with flexion of the scaphoid, 'signet-ring' sign. Gilula's lines [2] are disrupted. There is loss of overall height of the carpus, with the capitate being driven down between the scaphoid and triquetrum. No fracture is seen, and the remaining carpal bones are not dislocated as in a perilunate dislocation, which would result in a volarly dislocated lunate. The lunate lies in a flexed position (Fig. 2).

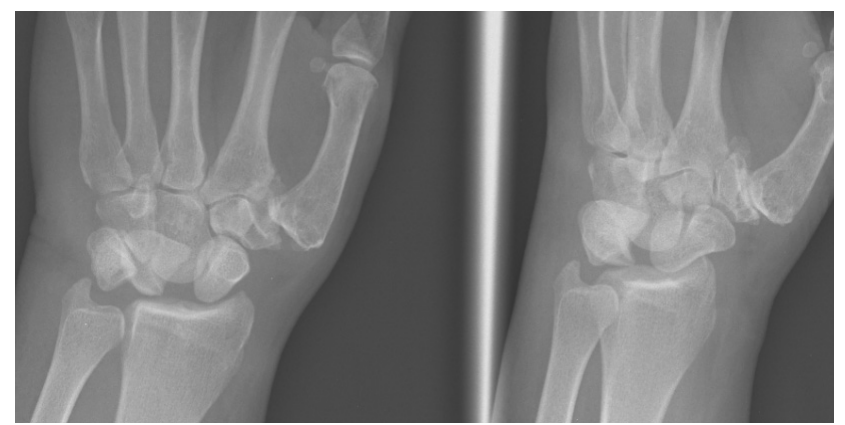

Fig. (1). Postero-anterior and oblique radiographs showing disruption of Gilula's lines and the scaphoid 'signet-ring' sign.

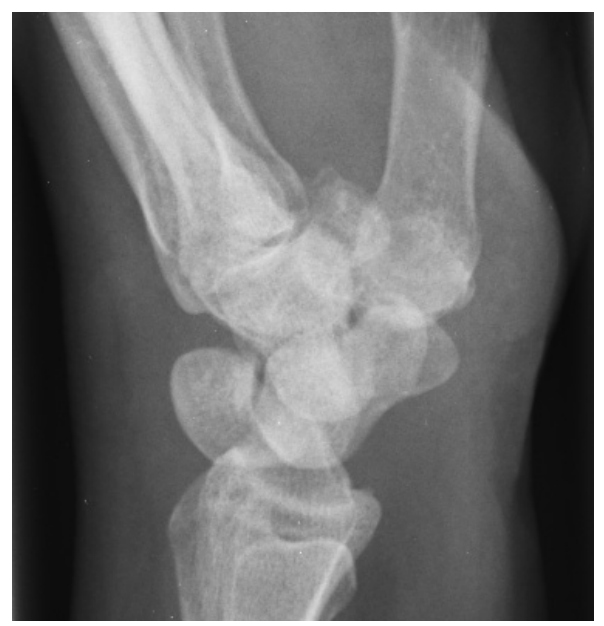

Fig. (2). Lateral radiograph clearly showing the dorsally dislocated lunate.

The patient was taken to the operating theatre and under general anaesthetic the lunate was reduced back into position. The lunate was easily reducible closed with simple pressure on the wrist dorsum, and stable with the wrist dorsiflexed and also in a neutral position as long as gentle traction was applied. This was confirmed on intra-operative $\mathrm{X}$-ray image intensifier dynamic screening. However, the lunate dislocated as soon as the wrist was brought to the neutral position without traction, and also with wrist palmarflexion.

The wrist was held in a neutral position with traction as the lunate was reduced. A single dorsally placed $1.6 \mathrm{~mm} \mathrm{~K}$ wire was used in a Kapandji fashion to buttress the lunate in position after reduction, and a below elbow Plaster of Paris cast was applied with the wrist in dorsiflexion. Postoperatively the position was confirmed radiographically (Fig. 3).
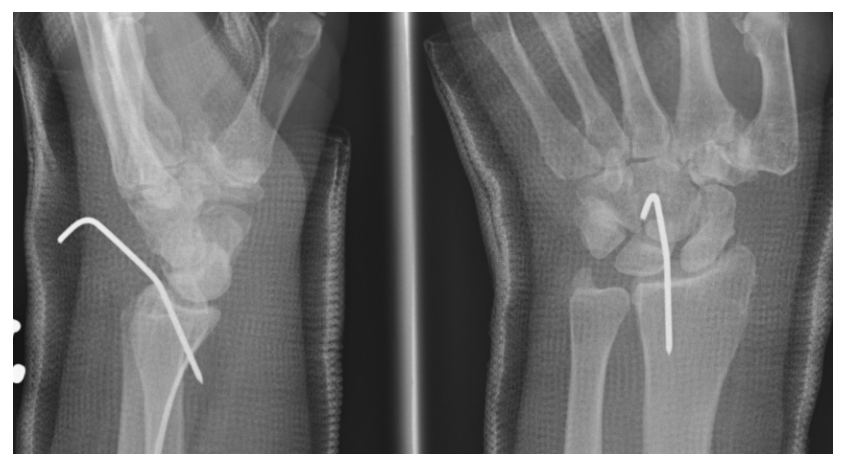

Fig. (3). Immediate post-operative radiographs showing lunate held reduced with a k-wire.

The K-wire was removed after two weeks and a Plaster of Paris cast was re-applied with the wrist still in dorsiflexion for a further week. Thus, the patient was kept for a total of three weeks in cast. Radiographs taken after cast removal show the lunate remained reduced (Fig. 4).

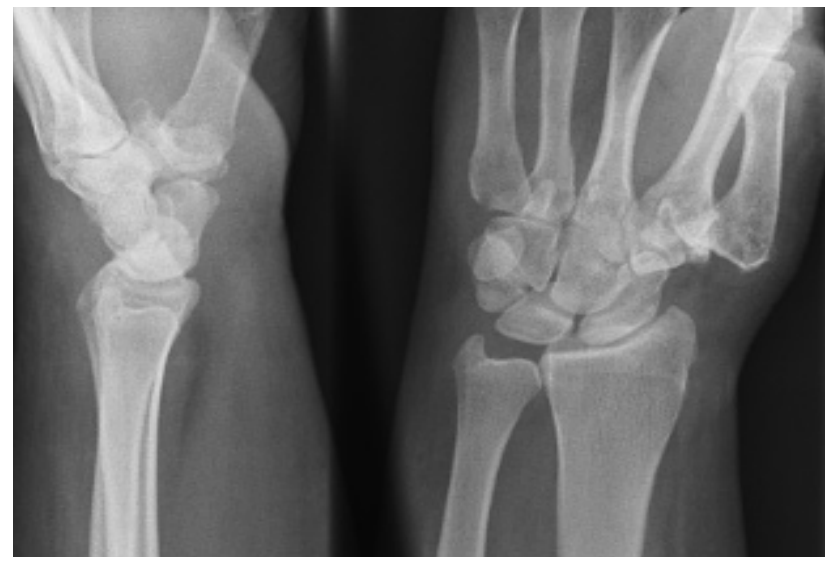

Fig. (4). Lunate reduced three weeks post-operatively: Gilula's three arcs have been restored.

On examination in clinic three weeks post-operatively there was no palpable mass dorsally as before, and it did not appear with passive or active wrist palmar-flexion which was possible to 30degrees. The patient complained of no pain around the wrist joint and only minimal stiffness. She was now able to fully pronate and supinate as compared to her unaffected side, with radial deviation improved by 5 degrees and ulnar deviation by 10 degrees, although she felt the wrist was still stiff. 
The patient was commenced on a hand physiotherapy regime which included full range of movement exercises, gradual strengthening, and proprioceptive training. She returned to partial duties at work successfully two weeks after removal of plaster cast, a total of five weeks after surgery. At the three month follow up she had a clinically stable wrist and reported she was able to carry out all her normal activities of daily living, with no difficulties at work.

\section{DISCUSSION}

The lunate is attached to several ligaments which are responsible for maintaining its position, particularly the radio-lunate, scapho-lunate, radio-scapho-lunate, and lunotriquetral ligaments. This is in addition to the surrounding carpal contours and radial ulnar facet providing added stability. The lunate is, therefore, very well supported in the normal individual and dislocation would require a large amount of energy to disrupt these structures.

Lunate dislocation is well described in the volar direction, usually as part of a peri-lunate carpal dislocation. In a perilunate dislocation the carpus dislocates dorsally as a whole, disrupting the stabilising ligaments, leaving the lunate behind in its lunate fossa. As the carpus then reduces back onto the radius the lunate can no longer remain keyed into its usual position and is forced volarly.

Mayfield [1] described sequential failure of the ligaments stabilising the lunate, culminating in complete dislocation. Four stages of increasing ligament injury were originally described. The first stage shows injury to the scapho-lunate and radio-scaphoid ligaments with scaphoid dislocation/ instability. Stage two involves capitate dislocation including "space of Poirier" disruption. Stage three has a dislocation of the triquetrum with radio-triquetral ligament rupture. The final stage reveals a complete failure of the radio-capitate, radio-triquetral, and dorsal radio-carpal ligaments. As well the lunate, failure of these ligaments may also lead to dislocation of other carpal bones such as the triquetrum. The Mayfield classification is commonly used to describe the mechanism of failure ligaments when planning reconstruction.

However, it is unclear whether the case we present follows the same pattern of disruption as the dislocation was dorsal rather than volar, and also the amount of energy involved was small, whereas the ligaments are very robust and would require a large amount of energy to injure. We presume that the pattern was not a mirror of a perilunate dislocation, i.e. that the carpus dislocated volarly and forced the lunate dorsally during reduction, as we know that the strength of the dorsal ligaments is such that a trivial injury could not cause them to fail.

In addition to the anatomical and biomechanical consideration, the early return to full function without formal exploration or reconstruction of the stabilising structures would suggest a different mechanism to any that we currently understand. There is the possibility of a preexisting weakness of the ligaments, but the patient had never previously described any significant or repetitive injury, and had not had any symptoms of instability.

As well as the resultant bony instability, volar lunate dislocation may also cause pressure and resultant dysfunction to the median nerve as it enters the carpal tunnel, and there are also reports of the ulnar nerve [3] being affected as a result of volar dislocation of the lunate.

Peri-lunate carpal dislocation may be treated with closed reduction, but may also require open reduction and formal reconstruction or repair of some or all of the injured ligaments in order to restore stability and allow return to function. In the acute setting with median nerve symptoms an emergency carpal tunnel decompression may be required. Carpal tunnel syndrome is often also described in delayed presentations of volar lunate dislocations. Herzberg [4] found 4 of 23 patients with perilunate dislocation presented with acute median nerve dysfunction. In another series Herzberg [5] reviewed 166 cases of perilunate dislocations with or without associated fracture, and noted that $25 \%$ of lunate dislocations are missed at initial presentation.

When assessing the position of the carpal bones on plain radiographs Gilula's lines [2] are a useful point to start from. Gilula studied the postero-anterior radiographs of over 90 patients with carpal fractures and fracture-dislocations and compared them with normal studies to establish a pattern of three smooth radiographic arcs. Disruption of one or more of these arcs suggests one or more carpal bones may be misaligned. When assessing our patient we can see that all three arcs have been disrupted. Following surgery the three arcs have been restored, with the articulating surfaces parallel again. The restraining ligaments would be in closest apposition with the carpus correctly aligned in order to heal.

Lunate dislocation and scapho-lunate ligament disruption have also been described with fracture of the scaphoid [6], thereby creating a disruption of the greater arc, with a characteristic transmission of energy across the intercarpal ligaments and exiting through the scaphoid creating a fracture. These are high energy injuries, as significant force such as a fall onto the outstretched hand would be required to fracture the scaphoid and disrupt the surrounding ligaments.

Overall carpal height may be estimated using the technique described by Youm et al. [7] by measuring the height of the carpus from the distal radius joint surface to the base of the middle finger metacarpal and comparison with the length of the middle metacarpal. This ratio is approximately $0.5(0.54+/-0.03)$. A reduction in this ratio would suggest a loss of carpal height.

To our knowledge there are two other reports in the English literature of isolated dorsal lunate dislocation without fracture, both of which were high energy injuries and required significant surgical procedures. Bilos and Hui [8] describe a case of repeated high energy injury in a boxer who dislocated the lunate dorsally. This required open reduction and multiple ligament reconstruction followed by $\mathrm{k}$-wire fixation and plaster immobilisation for two months. Seidenstein [9] reports a patient who fell from a truck with a forced hyper flexion injury as he fell, resulting in $75 \%$ subluxation of the lunate; a delay in diagnosis necessitated excision of the lunate.

There are similar reports of dorsal lunate dislocation with either concomitant fracture [10-12] or multiple tendon ruptures [13]. Bjerregaard et al. [10] describe a case of radial styloid fracture with associated dorsal dislocation of the 
lunate in a lorry driver who collided with a train. Neavin et al. [12] report an unrestrained passenger in a truck rollover accident who sustained dorsal lunate dislocation which was lying subcutaneously, as well as having fractures to the base of proximal phalanx of the fifth finger and comminuted intra-articular radial styloid fracture. Schwartz et al. [13] report an old dorsal dislocation of the lunate which required proximal row corpectomy and multiple tendon transfers.

This case has two features which have not been reported previously. Firstly, it is the result of a trivial injury. The injury sustained by our patient involved a relatively small amount of energy, less than that transmitted through a fall. The lunate was unstable when examined at the time of surgery, and we must assume ligamentous damage to some or all of the surrounding ligaments, as there was no associated fracture.

Secondly, this injury did not require open reduction and subsequent ligament repair, or other internal or external fixation, and has been treated with simple fixation with a single K-wire and plaster cast.

Treatment by closed reduction and k-wire fixation with only a short period of three weeks of immobilisation in a cast, with removal of the wire at two weeks, allowed early mobilisation of the wrist and has shown the lunate to remain reduced on follow-up radiographs.

Early mobilisation of wrist injuries has been shown to provide benefits to patients $[14,15]$ by speeding up the rate of functional recovery, minimising stiffness, swelling, and pain. Treatment with closed reduction and K-wire fixation results in minimal soft tissue violation. Immobilisation in a cast for only three weeks has the benefit of early mobilisation whilst restoring stability of the lunate, and subsequently allowing the patient an early return to work.

Patients reporting ongoing pain and swelling in the wrist after a trivial injury should be carefully examined, and in the case of isolated dorsal lunate dislocation should be considered for closed reduction and k-wire fixation, with early wire removal and mobilisation.

\section{CONFLICT OF INTEREST}

The authors confirm that this article content has no conflict of interest.

\section{ACKNOWLEDGEMENTS}

Declared none.

\section{REFERENCES}

[1] Mayfield JK. Mechanism of carpal injuries. Clin Orthop Rel Res 1980; 149: 45-54.

[2] Gilula LA. Carpal Injuries: Analytic approach and case exercises. Am J Roentgen 1979; 133: 503-17.

[3] Shariff Z, Uziogwe CE, Urso-Baiarda F, Shetty A. Ulnar nerve paresis secondary to lunate dislocation. Eur J Plast Surg 2009; 32: 333-5.

[4] Herzberg G, Forissier D. Acute dorsal trans-scaphoid perilunate fracture-dislocations: medium term results. J Hand Surg $\mathrm{Br} 2002$; 27(6): 498-502.

[5] Herzberg G, Comtet JJ, Linscheid RL, Amadio PC, Cooney WP Stalder J. Perilunate dislocations and fracture-dislocations: a multicenter study. J Hand Surg Am 1993; 18(5): 768-79.

[6] Komurcu M, Kurklu M, Ozturan KE, Mahirogullari M, Basbozkurt M. Early and delayed treatment of dorsal transscaphoid perilunate fracture-dislocations. J Orthop Trauma 2008; 22(8): 535-40.

[7] Youm Y, McMurtry RY, Flatt AE, Gillespie TE. Kinematics of the wrist: an experimental study of radial-ulnar deviation and flexionextension. J Bone Joint Surg 1978; 60-A (4): 423-31.

[8] Bilos ZJ, Hui PW. Dorsal dislocation of the lunate with carpal collapse. Report of two cases. J Bone Joint Surg 1981; 63-A (9): 1484-6.

[9] Seidenstein H. Two Unusual dislocations at the wrist. J Bone Joint Surg 1956; 38-A: 1137-41.

[10] Bjerregaard P, Holst-Nielsen F. Trans-styloid dorsal luxation of the lunate. Case report. Scand J Plast Reconstr Surg Hand Surg 1988; 22(3): 261-4

[11] Evans S. Radiological appearances of a dorsal fracture dislocation of the lunate. Radiogr Today 1991; 57(652): 30

[12] Neavin T, Lee WPA, Wollstein R. Dorsal dislocation of the lunate with distal radius fracture. Plastic Reconstruct Surg 2009; 124(6): 451e-2.

[13] Schwartz M G, Green S M, Coville F A. Dorsal dislocation of the lunate with multiple extensor tendon ruptures. J Hand Surg Am 1990; 15(1): 132-3.

[14] Dias JJ, Wray CC, Jones JM, Greg PJ. The value of early mobilisation in the treatment of Colles' fractures. J Bone Joint Surg (Br) 1987; 69-B (3): 463.

[15] McAuliffe TB, Hilliar KM, Coates CJ, Grange WJ. Early mobilisation of Colles' fractures. J Bone Joint Surg (Br) 1987; 69B (5): 727.

This is an open access article licensed under the terms of the Creative Commons Attribution Non-Commercial License (http://creativecommons.org/licenses/by-nc/3.0/) which permits unrestricted, non-commercial use, distribution and reproduction in any medium, provided the work is properly cited. 